\title{
Quantum strong coupling with protein vibrational modes
}

\author{
Robrecht M. A. Vergauwe ${ }^{1}$, Jino George ${ }^{1}$, Thibault Chervy ${ }^{a}$, James A. Hutchison ${ }^{a}$, Atef Shalabney ${ }^{2}$, \\ Vladimir Y. Torbeev ${ }^{1}$, Thomas W. Ebbesen ${ }^{a}$ \\ ${ }^{1}$ University of Strasbourg, CNRS, ISIS, 8 allée Gaspard Monge, 67000 Strasbourg, France \\ ${ }^{2}$ Department of physics and optical engineering, Ort Braude College, Karmiel, 21982 Israel
}

Poly-L-glutamic acid (sodium salt, nominal MW 15000-50000) and bovine serum albumin (lyophilized, > $99 \%$ ) were purchased from Sigma-Aldrich ( $\mathrm{GmbH}$, Germany). Deuteration of PLGA was performed with three dissolution-lyophilization cycles with $\mathrm{D}_{2} \mathrm{O}$ (99.9 atom $\% \mathrm{D}$, Sigma-Aldrich), BSA with two cycles. All samples were stored at $-20^{\circ} \mathrm{C}$. Just before usage, an aliquot was allowed to equilibrate at room temperature before dissolution with an appropriate amount of $\mathrm{D}_{2} \mathrm{O}$. All solution pD's were measured by determining the solution $\mathrm{pH}$ with a Mettler Toledo SevenCompact $\mathrm{pH}$ meter and applying the correction $\mathrm{pD}=\mathrm{pH}+0.4 .{ }^{1} \mathrm{NMR}$ spectra of $\mathrm{BSA}$ in $\mathrm{D}_{2} \mathrm{O}$ were measured on a $600 \mathrm{MHz}$ Bruker Avance III spectrometer.

Two optically polished circular ZnSe windows (Korth Kristalle GmbH, Germany, 25 mm diameter, 1 $\mathrm{mm}$ thickness) were coated with $10 \mathrm{~nm}$ of Au using electron-beam-assisted metal evaporation (ME300 PLASSYS, France; working pressure $\approx 10^{-5} \mathrm{mbar}$, deposition rate $\approx 0.2 \mathrm{~nm} / \mathrm{s}$ ). ${ }^{2}$ A planar FabryPérot cavity was assembled by interspacing a Mylar spacer ( $6 \mu \mathrm{m}$ thickness, Eurolabo SA, France) and a drop of protein solution between two of these mirrors and mounting this setup in a demountable liquid transmission cell holder (Harrick Scientific Inc., USA). ${ }^{2}$ All IR measurements were performed on a Nicolet 6700 FT-IR (Thermo-Fisher Inc., USA). For each spectrum, 256 inferograms were measured with a $0.964 \mathrm{~cm}^{-1}$ resolution. Transmission spectra of the bare molecules were measured in a similar fashion using uncoated ZnSe mirrors. Angle-dependent measurements were done by mounting the cell on a graded rotating stage (Thor labs Inc., USA). 
Extraction of the polariton peak positions was done by manually fitting of the polariton bands with a single peak, symmetric Gaussian model given by :

$$
f(x)=A \cdot e^{-\left(\frac{x-x_{o}}{B}\right)^{2}}+C
$$

where $A$ is the amplitude, $x_{0}$ the center position, $B$ determines the width and $C$ the offset. Fitting of the dispersion curves was done with the coupled oscillator model with either 1 or 2 independent excitons in order to retrieve the vacuum Rabi splitting energy. In these models, the polariton energies are given by the diagonalization of the respective coupling matrix ${ }^{3-6}$ :

$$
\left(\begin{array}{cc}
E_{c}\left(k_{/ /}\right) & \hbar \Omega_{R} / 2 \\
\hbar \Omega_{R} / 2 & E_{v i b}
\end{array}\right) \quad \text { and } \quad\left(\begin{array}{ccc}
E_{c}\left(k_{/ /}\right) & \hbar \Omega_{R, e x 1} / 2 & \hbar \Omega_{R, e x 2} / 2 \\
\hbar \Omega_{R, \text { ex } 1} / 2 & E_{v i b 1} & 0 \\
\hbar \Omega_{R, \text { ex } 2} / 2 & 0 & E_{v i b 2}
\end{array}\right)
$$

where $E_{c}\left(k_{/ /}\right)$is energy of the cavity mode as a function of the in-plane wave vector, $E_{v i b}, E_{v i b 1}$ and $E_{v i b 2}$ are the vibrational transition energies and $\hbar \Omega_{R}, \hbar \Omega_{R, v i b 1}$ and $\hbar \Omega_{R, v i b 2}$ are the vacuum Rabi splittings. The energy of the cavity mode as a function of the in-plane wave vector is given by $E_{c}=\frac{L_{c}}{\pi n_{c}} \cdot \sqrt{m^{2}+k_{/ /}^{2}}$, where $L_{c}$ is the cavity length, $n_{c}$ the cavity refractive index (here taken to be 1.45), $m$ the mode number of the mode to be coupled and $k_{/ /}$the in-plane wave vector. ${ }^{7}$ The in-plane wave vectors of the polariton peaks were calculated using the formula $k_{/ /}=$ $\frac{2 \pi}{k_{p}} \cdot n_{Z n S e} \cdot \sin \left(\theta_{Z n S e}\right)$, where $k_{p}$ is the polariton peak energy in wavenumbers, $n_{Z n S e}$ the mean refractive index of ZnSe at $1500-1700 \mathrm{~cm}^{-1}$ (here taken to be 1.9) and $\theta_{Z n S e}$ the incidence angle corrected for the refraction through the ZnSe windows. ${ }^{7}$ Calculation of the coupled cavity mode parameters (e.g. mode FWHM) was performed by extracting the free spectral range and cavity $Q$ factor from the peak positions and line widths of 10 higher order peaks located between 7000 and $4000 \mathrm{~cm}^{-1}$ via fitting to a Lorentzian lineshape model. The peak positon and FWHM of the PLGA $\gamma$ $\mathrm{COO}^{-}$and $\mathrm{BSA}$ amide $\mathrm{I}^{\prime}$ absorption band were determined by fitting respectively a Lorentzian and a Gaussian model. For the PLGA amide I' band, this needed to be done by visual inspection, i.e. without using fitting.

\section{REFERENCES}

(1) Glasoe, P. K.; Long, F. A. Use of Glass Electrodes To Measure Acidities in Deuterium Oxide. J. Phys. Chem. 1952, 64, 188-190. 
(2) George, J.; Shalabney, A.; Hutchison, J. A.; Genet, C.; Ebbesen, T. W. Liquid-Phase Vibrational Strong Coupling. J. Phys. Chem. Lett. 2015, 6, 1027-1031.

(3) Hakala, T. K.; Toppari, J. J.; Kuzyk, A.; Pettersson, M.; Tikkanen, H.; Kunttu, H.; Törmä, P. Vacuum Rabi Splitting and Strong-Coupling Dynamics for Surface-Plasmon Polaritons and Rhodamine 6G Molecules. Phys. Rev. Lett. 2009, 103, 053602.

(4) Bellessa, J.; Symonds, C.; Meynaud, C.; Plenet, J. C.; Cambril, E.; Miard, A.; Ferlazzo, L.; Lemaitre, A. Exciton/plasmon Polaritons in $\mathrm{GaAs} / \mathrm{Al}_{0.93} \mathrm{Ga}_{0.07} \mathrm{As}$ Heterostructures near a Metallic Layer. Phys. Rev. B: Condens. Matter Mater. Phys. 2008, 78, 2-5.

(5) Lidzey; Bradley; Armitage; Walker; Skolnick. Photon-Mediated Hybridization of Frenkel Excitons in Organic Semiconductor Microcavities. Science. 2000, 288, 1620-1623.

(6) Holmes, R. J.; Forrest, S. R. Strong Exciton-Photon Coupling in Organic Materials. Org. Electron. physics, Mater. Appl. 2007, 8, 77-93.

(7) Saleh, B. E. A.; Teich, M. C. Fundamentals of Photonics; John Wiley and Sons Inc.: Hoboken, USA; 2007.

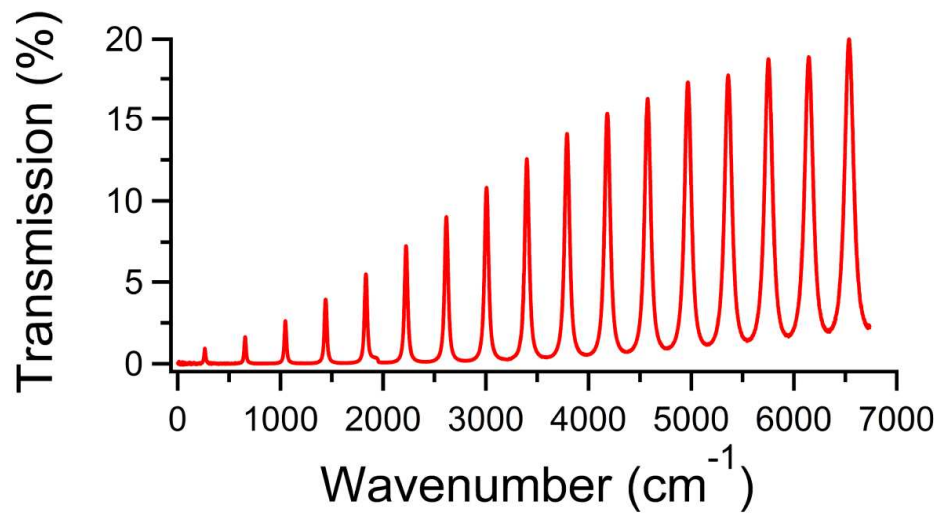

Figure S1. Transmission spectrum of an empty Fabry-Pérot cavity used for strong coupling of PLGA

and BSA. The cavity was constructed by sandwiching two $\mathrm{ZnSe}$ windows coated with $10 \mathrm{~nm}$ of $\mathrm{Au}$ with a polymeric spacer of $12 \mu \mathrm{m}$ nominal thickness. The free spectral range of this cavity was measured to be $392 \mathrm{~cm}^{-1}$ and the $Q$ factor 65.3 . 
A)

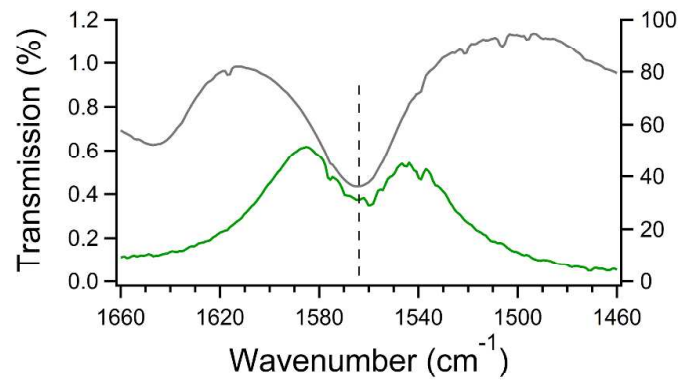

B)

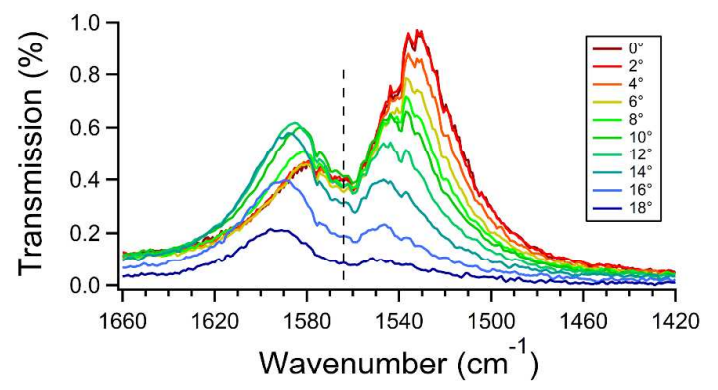

C)

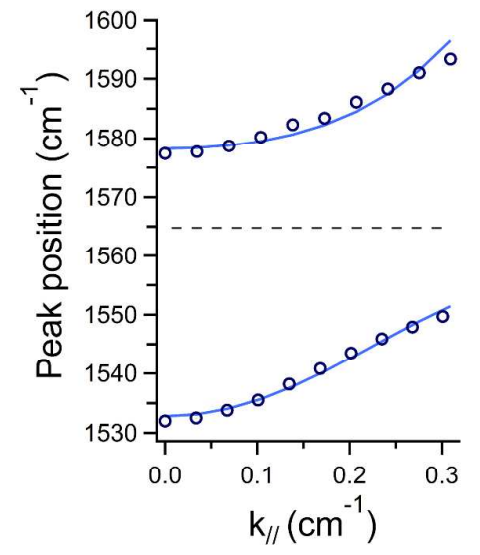

G)

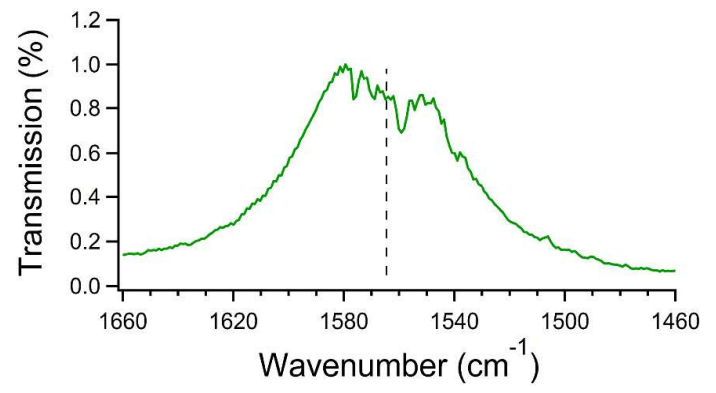

D)

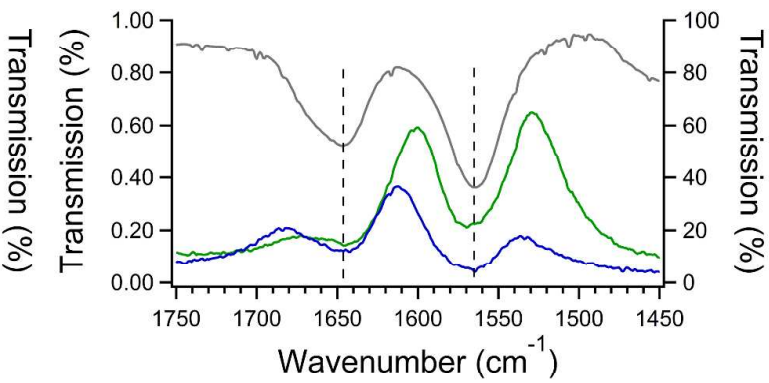

E)

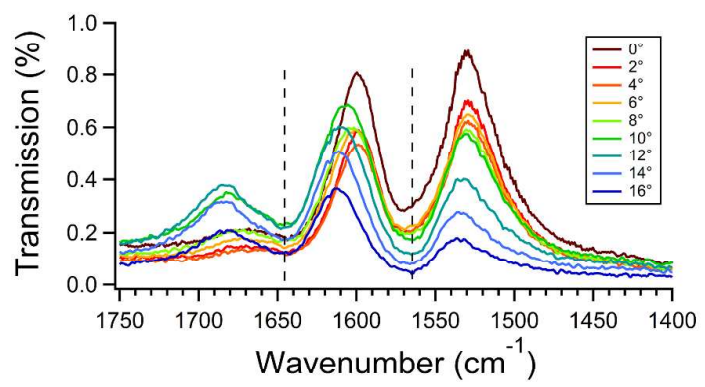

F)

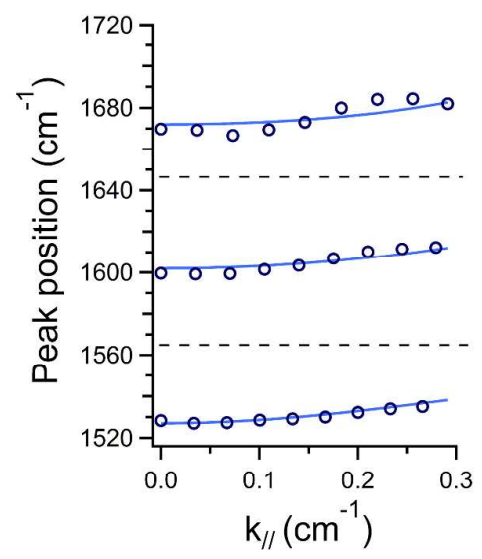

Figure S2. FTIR spectra and dispersion data of poly-L-glutamic acid. A-C) Data for PLGA at $2.5 \%$ (w/v). D-F) Data for PLGA at $10 \%(w / v) . ~ G)$ Data for PLGA at $1 \%(w / v) . A), D)$ \& G) IR transmission spectrum for cavity tuned close to either the amide $\mathrm{I}^{\prime}$ or $\gamma-\mathrm{COO}^{-}$asymmetric stretching vibration of PLGA. Green curves: resonance with the $\gamma$ - $\mathrm{COO}^{-}$side-chain asymmetric stretching mode. Blue curve: 
resonance with the amide I' mode. Grey curves: reference IR transmission spectrum of PLGA outside a cavity. B) \& E) Data of the angle-dependent dispersion measurements. C) \& F) Dispersion curves with the peak positions of the polariton branches as a function of the in-plane wave vector $k_{/ /}$(i.e. the component parallel to the mirror planes). The measured peak polariton peak positions are denoted with dark blue open circles and the coupled oscillator model fit with a light blue solid line. The location of the amide I' and $\gamma$ - $\mathrm{COO}^{-}$modes are marked with dashed lines. All measurements were performed in $\mathrm{D}_{2} \mathrm{O}$. 


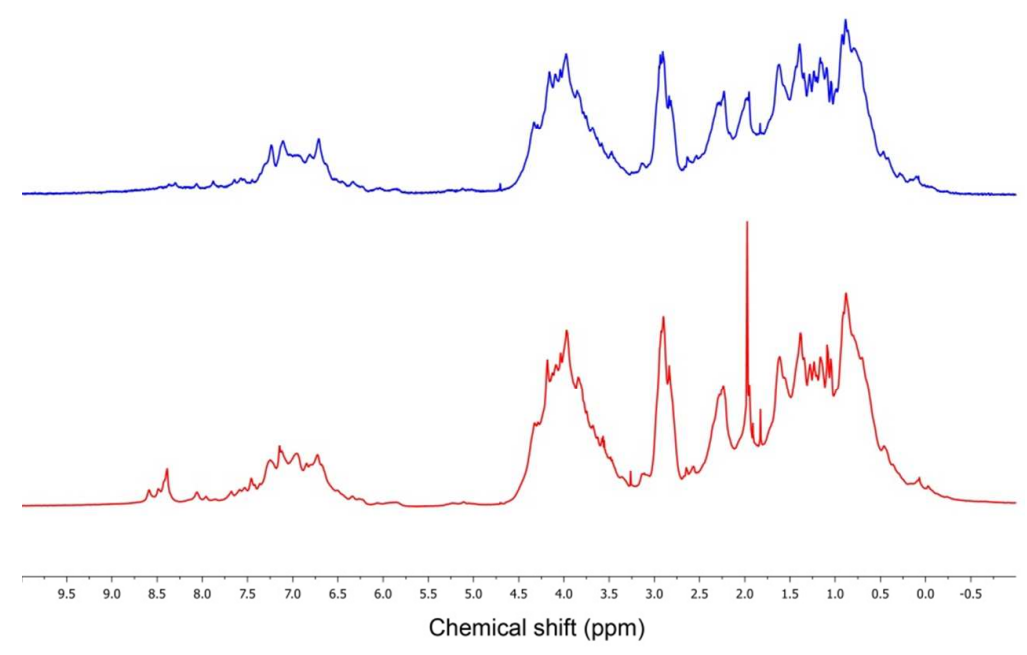

B)

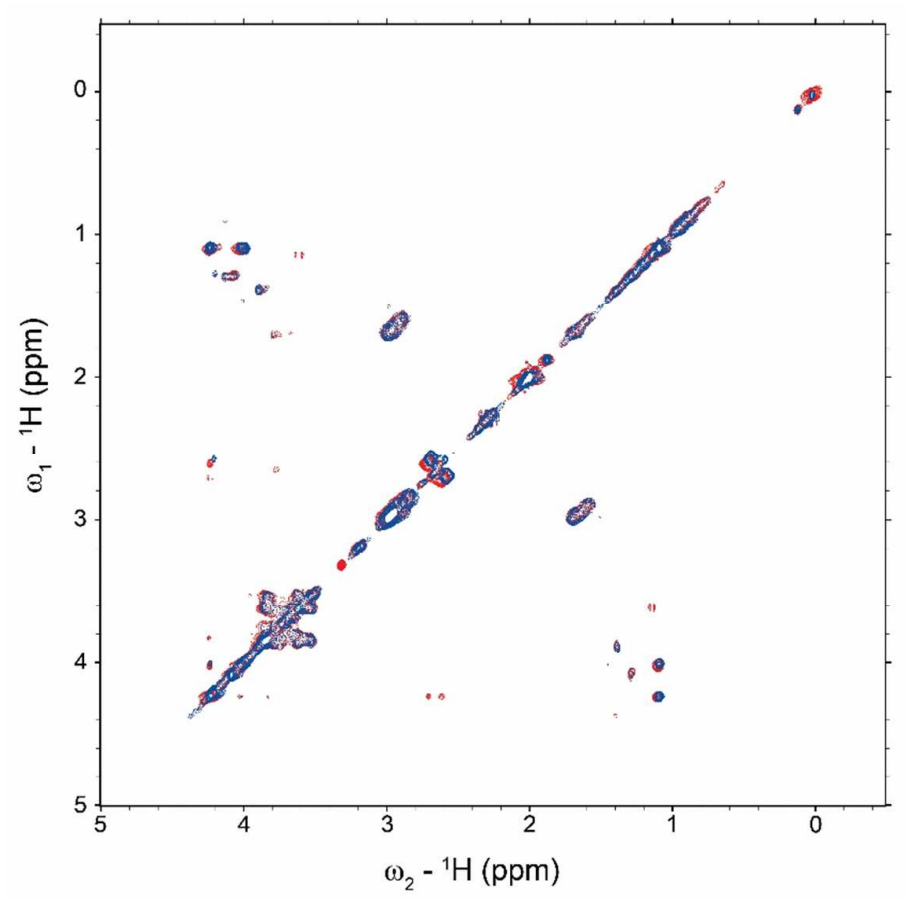

Figure S3. Fingerprint $600 \mathrm{MHz}{ }^{1} \mathrm{H}$ NMR spectra for BSA at two different concentrations. (A) $1 \mathrm{D}^{1} \mathrm{H}$ NMR for BSA at low concentration ( $c=1.6 \% \mathrm{w} / \mathrm{v}$; in blue) and at high concentration ( $\mathrm{c}=10 \% \mathrm{w} / \mathrm{v}$; in red). Amide signals are suppressed due to exchange with $\mathrm{D}_{2} \mathrm{O}$. Chemical shift range for $\mathrm{CH} \alpha$ protons ( 4.5-3.5 ppm) indicates predominantly $\alpha$-helical secondary structure. (B) Overlay of ${ }^{1} \mathrm{H}$-TOCSY (total correlation spectroscopy) spectra for the corresponding samples indicates that most of the structural features of BSA protein are preserved at two distinct concentrations. 
A)

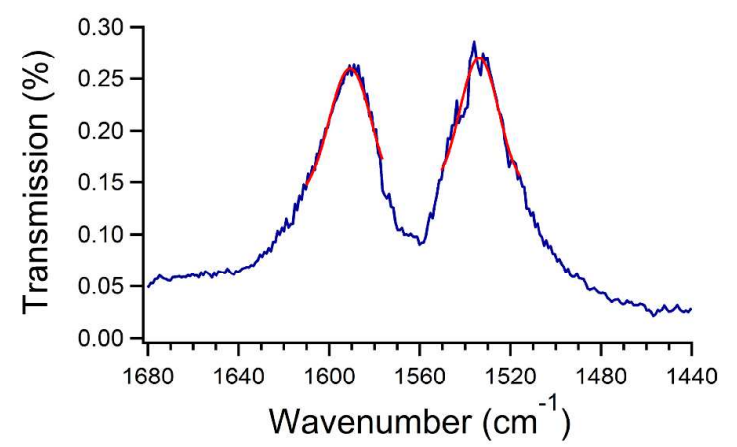

C)

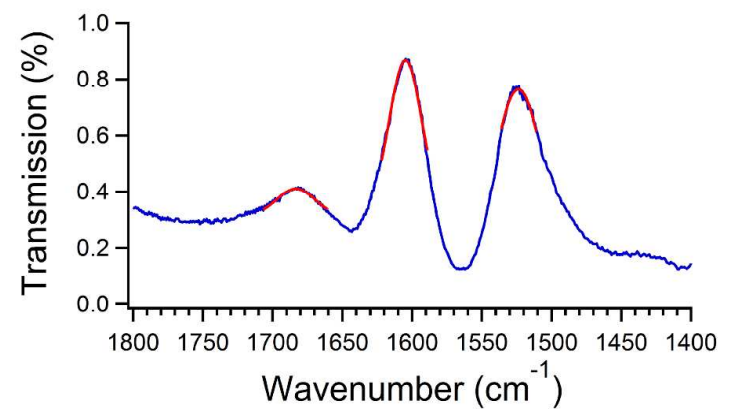

B)

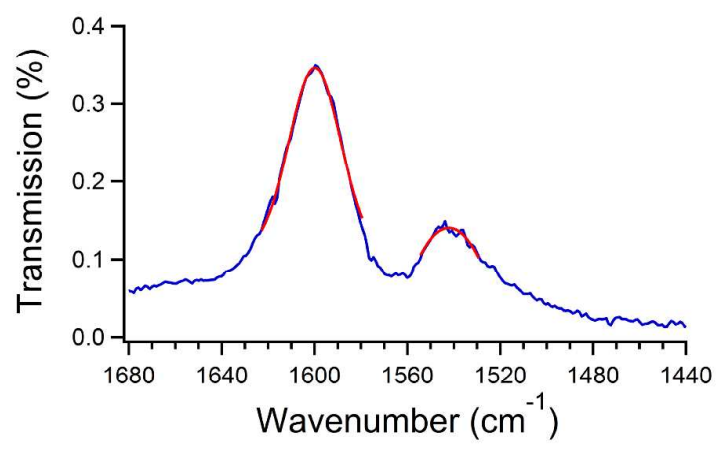

D)

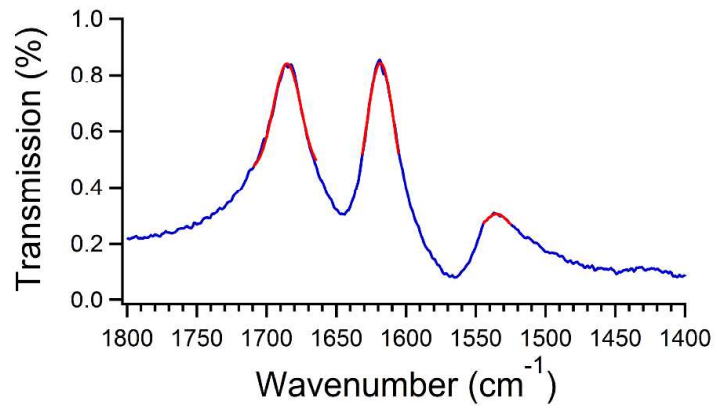

Figure S4. Peak extraction of the polariton peaks. Four examples of IR spectra of PLGA inside a resonant Faby-Perot cavity in which the polariton peaks have been fitted with a Gaussian model to determine the peak maximum position. A) PLGA 5\% $0^{\circ}$ angle of incidence. B) PLGA 5\% $10^{\circ}$ angle of incidence. C) PLGA $10 \% 0^{\circ}$ angle of incidence. D) PLGA $10 \% 10^{\circ}$ angle of incidence. Blue: Cavity IR spectra. Red: fit model. 